\title{
Frequência de suínos soropositivos para Salmonella sp.em granjas afetadas em diferentes níveis de severidade pela Síndrome Multissistêmica de Definhamento do Leitão Desmamado
}

\author{
Frequency of Salmonella seropositive pigs in farms affected by different severity levels \\ of the Post-weaning Multisystemic Wasting Syndrome \\ Patrícia Schwarz ${ }^{1}$, Jalusa Deon Kich ${ }^{2}$, Arlei Coldebella ${ }^{2}$, Leonardo Seyboth ${ }^{1}$, Cherlla Romeiro', \\ Luis Gustavo Corbellini ${ }^{3} \&$ Marisa Cardoso ${ }^{3}$
}

\begin{abstract}
Background: The Post-weaning Multisystemic Wasting Syndrome (PMWS) has caused considerable losses due to mortality and wasting of pigs and has been often associated with co-infections with other viral or bacterial agents. At the present time, PMWS is the most important infectious disease syndrome in the Brazilian swine production. With a variety of symptoms, this syndrome affects the immune system and can thus open the door for co-infections, which results in performance losses. Positive results of immunohistochemistry for PCV2 in pigs, we obtained the isolation of Salmonella enterica in 36.2\% cases, demonstrate that co-infection occurs in Brazilian herds. However, there is still determining whether there is an increase in the number of carriers of Salmonella sp. in swine herds affected by PCV2. From this, the present study aimed to investigate the frequency of animals positive for Salmonella sp. in pig herds with different levels of intensity of involvement by PMWS.

Material, Methods and Results: Productive performance data and most prevalent symptoms were investigated in herds associated to 11 Brazilian swine companies that reported the occurrence of PMWS. The production systems, where the presence of $S$. enterica in pigs had been previously confirmed, was selected. A three-site management system was adopted, with each stage (breeding, nursery and finishing) housed in separate sites. Herds $(\mathrm{n}=188)$ were classified according to the mortality and wasting frequency, and these data were considered as PMSW severity degrees. Blood samples were taken from slaughter pigs of each herd and submitted to an indirect ELISA test against Salmonella sp. Statistical analysis was performed using chi-square for linear trend to test the hypothesis of increased proportion of flocks positive for Salmonella with levels of involvement of PMWS, to increase the power of the sample, the variable Salmonella infection was collapsed into d" $70 \%$ and> 70\%. The analysis was performed using the software EpiInfo 6.0.

Discussion: The presence of clinical signs consistent with PMWS in herds, coupled with the confirmation from the occurrence of PCV2 in farms associated with the company, was observed in all farms included in the study. The intensity of infection has been associated with increased culling and mortality in herds affected and, therefore, was adopted as a criterion level of involvement of clinical PMWS. In this study the factor was not considered a medical illness, but the level of seroprevalence of Salmonella sp., a rate that has been used as an indicator of prior exposure to this agent and that is a risk factor for the presence of carrier animals asymptomatic slaughter and the contamination of carcasses. Thus, the results indicate that farms that had involvement of more severe PMWS were classified in the prevalence of high-risk $(>70 \%)$ for the presence of carriers of Salmonella sp. slaughter. In all companies a decrease on the animal performance was detected and a tendency of a higher frequency of enteric symptoms associated to PMWS was observed. Seroprevalence $>70 \%$ against Salmonella was associated to higher PMWS severity ( $\mathrm{P}=0.003$ ). This result may be related to the immune impairment caused by PMWS that may predispose to Salmonella infection, as well as to the occurrence of common risk factors to both infections in affected herds. In conclusion, a high frequency of Salmonella carrier pigs may be an additional problem in farms severely affected by PMWS.
\end{abstract}

Keywords: swine, PMWS, PCV2, Salmonella seroprevalence.

Descritores: suíno, Síndrome Multissistêmica do Leitão Desmamado, PCV2, soroprevalência de Salmonella. 
Schwarz P., Kich J.D., Coldebella A., Seyboth L., Romeiro C., Corbellini L.G. \& Cardoso M. 2010. Frequência de suínos soropositivos para Salmonella sp. em granjas afetadas em diferentes níveis... Acta Scientiae Veterinariae. $38(2): 127-132$.

\section{INTRODUÇÃO}

O circovírus suíno tipo 2 (PCV2) causa a Síndrome Multissistêmica de Definhamento do Leitão Desmamado (SMDLD), uma das doenças mais importantes para a suinocultura brasileira na atualidade $[7,8]$. Os prejuízos causados pelo PCV2 decorrem dos piores índices produtivos do rebanho, do aumento do uso de antimicrobianos e das condenações de carcaças $[2,3,9,11,12]$. As manifestações da SMDLD compreendem sintomas respiratórios, entéricos, reprodutivos e a refugagem $[2,6,9,11]$. Ao lado disso, a infecção pelo PCV2 compromete o sistema imune e predispõe a coinfecções virais e bacterianas $[2,3,9]$.

Salmonella enterica é uma das principais causas de doenças transmitidas por alimentos de origem animal [16]. A infecção pela maioria dos sorovares não resulta em sinais clínicos evidentes nos suínos portadores [20]. Por essa razão, o controle tem sido baseado no monitoramento dos níveis de soroprevalência dos rebanhos e adoção de medidas que diminuam o número de portadores, considerados como fator de risco para a contaminação de carcaças [1]. Em suínos, o status imune do animal pode influenciar o curso da infecção [20], o que leva a supor que a coinfecção com o PCV2 no rebanho pode ser um fator associado com o aumento de portadores. Em suínos positivos na imuno-histoquímica para PCV2, obteve-se isolamento de Salmonella enterica em $36,2 \%$ dos casos, demonstrando que a coinfecção ocorre nos rebanhos brasileiros [23]. Entretanto, ainda resta investigar se há um incremento do número de portadores de Salmonella sp. em rebanhos suínos acometidos pelo PCV2.

A partir disso, o presente estudo visou a determinar a frequência de animais soropositivos para Salmonella sp. em rebanhos suínos com diferentes níveis de intensidade de acometimento pelo SMDLD.

\section{MATERIAIS E MÉTODOS}

Este estudo foi conduzido em granjas de 13 empresas das regiões Sul, Sudeste e Centro-oeste do Brasil, no período de janeiro de 2007 a agosto de 2008. De cada empresa, foram investigadas de $3 \%$ a $10 \%$ das granjas de crescimento e terminação, totalizando 188 granjas.

Primeiramente, foi conduzida uma investigação da situação em relação ao SMDLD em cada empresa. Para tanto, foram coletados dados referentes: aos gastos com medicamentos, resultados produtivos e econômicos da atividade, status sanitário dos rebanhos, ocorrência de PCV2 associada à mortalidade e refugagem, sintomas mais frequentemente encontrados, resultados dos principais parâmetros produtivos e uso de antimicrobianos. Os dados referentes a índices zootécnicos, como conversão alimentar (CA), ganho de peso diário (GPD), níveis de mortalidade, refugagem e gastos com antimicrobianos foram informados pelo gerenciamento técnico das empresas. A ocorrência de síndromes entéricas e respiratórias foi pesquisada no banco de dados das empresas participantes do estudo num período retrospectivo de um ano.

Os rebanhos investigados apresentavam sinais clínicos compatíveis com SMDLD (refugagem, apatia, tosse, diarreia e/ou lesões cutâneas) e foram classificados em níveis clínicos de acometimento pelo SMDLD (Tabela 1), considerando os índices de refugagem e mortalidade como indicadores. Todos os rebanhos eram associados a empresas que tinham a ocorrência de SMDLD confirmada por exames histológicos e imuno histoquímica, conforme descrito anteriormente [8]. Para a determinação de frequência de animais soropositivos para Salmonella sp., foi realizada colheita de sangue de 30 animais abatidos de cada uma das 188 granjas, perfazendo 5.640 amostras analisadas pelo teste de ELISA-LPS desenvolvido com antígeno somático do sorovar Typhimurium [14]. Os lotes investigados foram classificados em níveis de soroprevalência $\leq 40 \%$, entre $40 \%$ e $70 \%$ e $>70 \%$, conforme critério de classificação proposto anteriormente [17], onde o grupo de maior soroprevalência é considerado como o de maior risco para a contaminação de carcaças na linha de abate.

A análise estatística foi realizada através do Qui-quadrado de tendência linear para testar a hipótese do aumento da proporção de rebanhos positivos para Salmonella com relação aos níveis de acometimento da SMDLD; para aumentar o poder da amostra, a variável soroprevalência para Salmonella foi dicotomizada em $\leq 70 \%$ e $>70 \%$. A análise foi realizada utilizando o software EpiInfo 6.0 [10].

\section{RESULTADOS}

Das 13 empresas investigadas, 11 identificaram o circovírus suíno como importante obstáculo para a otimização do desempenho produtivo dos re- 
banhos, indicando o aumento da mortalidade, refugagem e gastos com medicamentos como as variáveis de maior impacto para os prejuízos produtivos. Os custos adicionais estimados por animal, com a entrada da SMDLD, variaram entre US\$ 1.90 e US\$ 5.20 por animal terminado, não incluindo prejuízos adicionais na industrialização de produtos cárneos pelo matadouro frigorífico. $\mathrm{O}$ aumento de gastos com antimicrobianos foi bastante variável entre unidades produtivas, sendo o grupo dos macrolídeos o mais utilizado em todas as empresas estudadas. Os parâmetros produtivos médios, mortalidade, conversão alimentar (CA) e ganho de peso diário (GPD), após o acometimento dos rebanhos pela SMDLD foram piores comparados com os dados históricos médios das empresas (Tabela 2).

Os sintomas entéricos foram mais frequentemente associadas ao SMDLD em oito das 13 empre- sas, com uma frequência média de $62 \%$ para manifestações entéricas e de $38 \%$ para respiratórias. Em quatro empresas foram identificados sintomas respiratórios como os de maior ocorrência nos rebanhos acometidos por PCV2, com uma frequência de 59\% para manifestação respiratória e de $41 \%$ para entéricas. Uma ocorrência idêntica de ambas as manifestações clínicas foi identificada em uma empresa incluída neste estudo.

Das 188 granjas em que foram colhidas amostras para determinar a frequência de animais soropositivos para Salmonella sp., 35 (18,62\%) tinham soroprevalência $<40 \%$; $11(5,85 \%)$, soroprevalência entre $40 \%$ e $70 \%$ e 142 (75,53\%), mais de $70 \%$ de soropositivos. Em relação ao nível de severidade de SMDLD, 32 (17,02\%) foram classificadas como nível 1, $54(28,73 \%)$ no nível 2 e $102(54,25 \%)$ no nível 3 (Tabela 3). A proporção de rebanhos com

Tabela 1. Critérios para classificação das granjas em níveis de intensidade de acomentimento pela Síndrome Multissistêmica do Definhamento do Leitão Desmamado.

\section{Mortalidade $\quad$ Refugagem}

Nível 1

Nível 2

Nível 3 até $2 \%$

2 a $3 \%$

acima de $3 \%$ até $2 \%$

2 a $4 \%$

Tabela 2. Parâmetros produtivos médios após o acometimento dos rebanhos pela Síndrome Multissistêmica do Definhamento do Leitão Desmamado, comparados com os dados históricos em 11 empresas produtoras de suínos no Brasil, no período de janeiro/2007 a dezembro/2008.

\begin{tabular}{cccc}
\hline & Dados históricos & \multicolumn{2}{c}{ Após SMDLD } \\
\cline { 2 - 4 } Parâmetro & Média & Média & Mínimo/Máximo \\
\hline $\begin{array}{c}\text { Mortalidade (\%) } \\
\begin{array}{c}\text { Conversão Alimentar } \\
(\mathrm{kg} / \mathrm{kg})\end{array}\end{array}$ & 1,6 & 4,9 & $2,5-10,0$ \\
\begin{tabular}{c} 
Ganho de Peso Diário $(\mathrm{g})$ \\
\hline
\end{tabular} & 824 & 2,9 & $2,3-3,3$ \\
\hline
\end{tabular}


prevalência maior do que $70 \%$ aumentou de forma significativa com o aumento dos níveis de severidade de SMDLD $(\mathrm{P}=0,003)$ quando comparados com o nível 1.

\section{DISCUSSÃo}

Na maioria (11/13) das empresas, o setor de gerenciamento técnico apontou a circovirose como o mais grave problema sanitário que afeta o setor, devido ao incremento nos custos de produção dos suínos, afetando diretamente a rentabilidade e a competitividade. Essa percepção de profissionais que atuam no meio produtivo concorda com a avaliação da comunidade científica, que classifica a circovirose suína como a doença mais importante da produção de suínos na atualidade, sendo este conjunto de síndromes diagnosticada em diversas regiões brasileiras e em todo mundo $[3,7,19,21]$. As perdas econômicas estão associadas com o decréscimo de performance de parâmetros produtivos como CA e GPD, utilizados comumente como referência para custos de produção no mercado produtor de suínos. No presente estudo, observa-se uma nítida piora desses índices em relação aos dados históricos das empresas que apontaram o PCV2 como problema sanitário. Especificamente, os índices de mortalidade sofreram um incremento significativo, o que está de acordo com dados de outros países que relatam a ocorrência da infecção pelo PCV2 [9,19]. O custo da SMDLD é bastante significativo para o retorno financeiro da atividade, sendo responsável por perdas avaliadas em até U\$ 6.00 por suíno entregue ao abate
[9]. No presente estudo, as perdas estimadas ficaram abaixo dessa estimativa, porém não foram avaliados dados de falta de uniformidade de lotes e condenação de carcaças ao abate, o que leva a supor que os prejuízos decorrentes da infecção por PCV2 nessas empresas tenham alcançado níveis ainda mais elevados.

O aumento de custos em plantéis positivos para SMDLD também é decorrente do aumento da ocorrência de infecção por outros agentes associados ao PCV2. Observa-se uma tendência de predomínio de sintomas respiratórios ou entéricos associados à infecção pelo PCV2, de acordo com a empresa avaliada, indicando que infecções já existentes no rebanho podem recrudescer durante a ocorrência de SMDLD. Ao lado disso, o próprio PCV2 pode causar as lesões observadas no trato respiratório e digestório $[9,8,23]$. A ocorrência de infecções bacterianas secundárias também explica o aumento do uso de antimicrobianos detectado nas unidades produtivas avaliadas, principalmente de macrolídeos, um princípio ativo frequentemente utilizado de forma metafilática em infecções bacterianas na suinocultura [4].

A presença de sinais clínicos compatíveis com SMDLD nos rebanhos, associado à confirmação prévia da ocorrência do PCV2 em granjas associadas à empresa, foi observada em todas as granjas incluídas no estudo. A intensidade da infecção tem sido associada com o aumento da refugagem e da mortalidade nos rebanhos acometidos $[7,8,12,19]$ e, por essa razão, foi adotado como critério de nível de acometimento clínico do SMDLD. A associação desses níveis e a soroprevalência de Salmonella sp. nos re-

Tabela 3. Distribuição de 188 granjas brasileiras com diferentes níveis de intensidade de acometimento pela Síndrome Multissistêmica do Definhamento do Leitão Desmamado de acordo com a soroprevalência para Salmonella sp., no período de janeiro/2007 a dezembro/2008.

Nível de acometimento pelo SMDLD
Número de rebanhos

\section{Soroprevalência para Salmonella sp.}

$(\%)$

\begin{tabular}{cccc}
\hline & & $>\mathbf{7 0 \%}$ & $\leq \mathbf{7 0 \%}$ \\
\cline { 3 - 4 } Nível 1 & 32 & $15(46,9 \%)$ & $17(53,15 \%)$ \\
Nível 2 & 54 & $46(85,2 \%)$ & $8(14,8 \%)$ \\
Nível 3 & 102 & $81(79,4 \%)$ & $21(20,6 \%)$ \\
\hline
\end{tabular}


banhos foi observada, estando de acordo com relato prévio da ocorrência de coinfecção de Salmonella sp. e do PCV2 em suínos com lesões entéricas provenientes de granjas do Rio Grande do Sul [23]. No presente estudo, o fator considerado não foi a doença clínica, mas o nível de soroprevalência para Salmonella sp., índice que tem sido empregado como um indicador de exposição prévia a esse agente e que constitui um fator de risco para a presença de animais portadores assintomáticos ao abate e para a contaminação de carcaças $[1,17]$. Dessa forma, os resultados obtidos indicam que granjas que apresentavam acometimento de SMDLD mais severo foram classificadas no nível de soroprevalência de maior risco $(>70 \%)$ para a presença de portadores de Salmonella sp. ao abate.

Essa observação pode estar relacionada ao fato de rebanhos com maior acometimento pelo SMDLD apresentarem um comprometimento mais intenso do sistema imune, predispondo os animais à infecção por microorganismos presentes no ambiente da granja. Estudos têm demonstrado que a contaminação residual de creches e granjas de terminação com Salmonella sp. ocorre no nosso país [18, 22]. Dessa forma, uma eventual imunossupressão pela infecção prévia pelo PCV2 em suínos alojados em ambientes contaminados poderia resultar em maior suscetibilidade à infecção e maior nível de soroprevalência ao abate. Associação semelhante foi anteriormente observada na França, com o Vírus da Síndrome Respiratória e Reprodutiva dos Suínos (PRSS), onde um maior risco de soroconversão para Salmonella esteve relacionado com o nível de soroprevalência de PRSS no rebanho, provavelmente devido ao quadro de imunossupressão observado em decorrência da infecção viral [5].
Entretanto, é preciso considerar que existem vários fatores de risco em comum para a ocorrência de Salmonella sp. e de SMDLD em rebanhos suínos, indicando que a presença dos mesmos na granja pode determinar uma maior probabilidade de infecção para ambos os agentes. Entre esses fatores, destacam-se o tamanho do lote, a mistura de animais de diversas origens e a higiene das instalações $[5,13,15,19]$. A presença desses fatores implica na maior mistura de animais, resultando em grupos heterogêneos e no intercâmbio de microorganismos patogênicos dentro dos lotes formados. $\mathrm{O}$ aumento do estresse dos animais, decorrente do alojamento, associado à presença residual de Salmonella sp. no ambiente, poderia determinar um maior nível de infecção para ambos os agentes nos rebanhos. Por outro lado, o aumento das infecções bac-terianas secundárias decorrentes da imunodepressão causada pelo PCV2, leva ao incremento do uso de antimicrobianos via ração, o que altera a microbiota intestinal e pode favorecer a colonização por Salmonella sp. [5].

A partir desses resultados, é possível supor que medidas que contribuam para a diminuição da intensidade de acometimento pelo SMDLD no rebanho poderão ter um impacto igualmente favorável na frequência de animais portadores de Salmonella sp.

\section{CONCLUSÃO}

A severidade de acometimento clínico pela Síndrome Multissistêmica de Definhamento do Leitão Desmamado pode estar associada com o incremento na frequência de portadores de Salmonella sp. em rebanhos suínos.

\section{REFERÊNCIAS}

1 Alban L. \& Stärk K.D.C. 2005. Where should the efforts be put to reduce the Salmonella presence in the slaughtered swine carcass effectively? Preventive Veterinary Medicine. 68(1): 63-79.

2 Allan G.M. \& Ellis J. 2000. Porcine circovirus: A review. Journal of Veterinary Diagnostic Investigation. 12(1): 3-14.

3 Allan G.M., McNeilly F., Kennedy S., Daft B., Clarck E.G., Ellis J.A., Haines D.M., Meehan B. \& Adarr B.M. 1998. Isolation of porcine circovirus-like virus from pigs with wasting disease in the USA and Europe. Journal of Veterinary Diagnostic Investigation. 10(1): 3-10.

4 Barcellos D.E.S.N. \& Sobestiansky J. 1998. Uso de antimicrobianos em suinocultura. Goiânia: Art3 Impressos Especiais, $108 \mathrm{p}$.

5 Beloeil P.A., Chauvin C., Proux K., Fablet C., Madec F. \& Alioum A. 2007. Risk factors for Salmonella seroconversion of fattening pigs in farrow-to finish herds. Veterinary Research. 38(6): 835-848. 
6 Bolin S.R., Stoffrengen W.C., Nayar G.P. \& Hamel A.L. 2001. Postweaning multisystemic wasting induced after experimental inoculation of cesarean-derived, colostrum-deprived piglets with type 2 circovirus. Journal of Veterinary Diagnostic Investigation. 13(3): 185-194.

7 Ciacci-Zanella J.R. \& Mores N. 2003. Diagnosis of post-weaning multisystemic wasting syndrome in pigs in Brazil caused by porcine circovirus type 2. Arquivo Brasileiro de Medicina Veterinária e Zootecnia. 55(5): 522-527.

8 Corrêa A.M.R., Pescador C.A., Schmitz M., Zlotowsky P., Rozza D.B., Oliveira E.C., Barcellos D.E. \& Driemeier D. 2006. Aspectos clínico-patológicos associados à circovirose suína no Rio Grande do Sul. Pesquisa Veterinária Brasileira. 26(1): 9-13.

9 Ellis J.A., Bratanich A., Clarck E.G, Allan G., Meehan B., Haines D.M., Harding J., West K.H., Krakowka S., Konoby C., Hassard L., Martin K. \& McNelly F. 2000. Coinfection by porcine circoviruses and porcine parvovirus in pigs with naturally acquired post-weaning multisystemic wasting syndrome. Journal of Veterinary Diagnostic Investigation. 12(1): 21-27.

10 Epi-Info 6.0. Center of Disease Control. www.cdc.gov/EpiInfo.

11 Harding J.C. 2007. Status of porcine circovirus diseases in Western Canada. Canadian Veterinary Journal. 48(3): 267268.

12 Harding J.C. 2004. The clinical expression and emergence of porcine circovirus 2. Veterinary Microbiology. 98(2): 131135 .

13 Kich J. D., Mores N., Piffer I., Coldebella A., Amaral A., Ramminger L. \& Cardoso M. 2005. Fatores de risco associados com a prevalência sorológica de Salmonella em granjas comerciais de suínos no sul do Brasil. Ciência Rural. 35(2): 398405 .

14 Kich J.D., Schwarz P., Silva L.E., Coldebella A., Piffer I.A., Vizzoto R. \& Cardoso M. 2007. Development and application of an enzyme-linked immunosorbent assay to detect antibodies against prevalent Salmonella serovars in swine in southern Brazil. Journal of Veterinary Diagnostic Investigation. 19(3): 510-517.

15 Lo Fo Wong D.M.A., Dahl J., Stege H., van der Wolf P.J., Leontides L., von AltrockA. \& Thorberg B.M. 2004. Herd-level risk factors for subclinical Salmonella infection in European finishing-pig herds. Preventive Veterinary Medicine. 62(4): 253-266.

16 Mead P.S., Slutsker L., Dietz V., McCaig L.F., Bresee J.S., Shapiro C., Griffin P.M. \& Tauxe R.V., 1999. Food-related illness and death in the United States. Emerging Infectious Diseases. 5(5): 607-625.

17 Mousing J., Jensen P. T., Halgaard C., Bager F., Feld N., Nielsen B., Nielsen J. P. \& Bech-Nielsen S. 1997. Nation-wide Salmonella enterica surveillance and control in Danish slaughter swine herds. Preventive Veterinary Medicine. 29(4): 247-61.

18 Müller M., Schwarz P., Kich J. \& Cardoso M. 2009. Perfil sorológico e de isolamento de Salmonella em suínos no início da terminação e ao abate. Ciência Animal Brasileira. 10(3): 931-937.

19 Rose, N., Larour G., Le Diguerher G, Eveno E., Jolly J.P., Blanchard P., OgerA., Le Dimma M., JestinA. \& Madec F. 2003. Risk factors for porcine post-weaning multisystemic wasting syndrome (PMWS) in 149 French farrow-to-finishing herds. Preventive Veterinary Medicine. 61(3): 209-225.

20 Schwartz K.J. 1991. Salmonellosis in Swine. Continuing Education 13(2): 202-209.

21 Segales J., Sitjar M., Domingo M., Dee S., Del Pozo M., Noval R., Sacristan C., De La Heras M., Ferro A. \& Latimer S. 1997. First report of post-weaning multisystemic wasting syndrome in pigs in Spain. Veterinary Records. 141(23): 600601.

22 Silva L.E., Gotardi C.P., Vizzoto R., Kich J. \& Cardoso M. 2006. Infecção por Salmonella enterica em suínos criados em um sistema integrado de produção do sul do Brasil. Arquivo Brasileiro de Medicina Veterinária Zootecnia. 58(4): 455461.

23 Zlotowski P., CorrêaA.M.R., Barcello D.E.B., Cardoso M., Vasconcelos A.C., Castro L.A. \& Driemeier D. 2009. Necrotic enterocolitis in pigs naturally infected by porcine circovirus type 2. Ciência Rural. 39(6): 1801-1807. 